\title{
Neutral Fitness Landscape in the Cellular Automata Majority Problem
}

\author{
S. Verel ${ }^{1}$, P. Collard ${ }^{1}$, M. Tomassini ${ }^{2}$, and L. Vanneschi ${ }^{3}$ \\ 1 Université de Nice-Sophia Antipolis/CNRS (verel,pc)@i3s.unice.fr \\ ${ }^{2}$ University of Lausanne Marco.Tomassini@unil.ch \\ 3 University of Milano vanneschi@disco.unimib.it
}

\begin{abstract}
We study in detail the fitness landscape of a difficult cellular automata computational task: the majority problem. Our results show why this problem landscape is so hard to search, and we quantify the large degree of neutrality found in various ways. We show that a particular subspace of the solution space, called the "Olympus", is where good solutions concentrate, and give measures to quantitatively characterize this subspace.
\end{abstract}

\section{Introduction}

Cellular automata (CAs) are discrete dynamical systems that have been studied for years due to their architectural simplicity and the wide spectrum of behaviors they are capable of [1]. Here we study CAs that can be said to perform a simple "computational" task. One such task is the so-called majority or density task in which a two-state CA is to decide whether the initial state contains more zeros than ones or vice versa. In spite of its apparent simplicity, it is a difficult problem for a $\mathrm{CA}$ as it requires a coordination among the automata. As such, it is a perfect paradigm of the phenomenon of emergence in complex systems. That is, the task solution is an emergent global property of a system of locally interacting agents. Indeed, it has been proved that no CA can perform the task perfectly i.e., for any possible initial binary configuration of states [2]. However, several efficient CAs for the density task have been found either by hand or by using heuristic methods, especially evolutionary computation [3, 4 [5]. For a recent review see [6].

All previous investigations have empirically shown that finding good CAs for the majority task is very hard. However, there have been no investigations, to our knowledge, of the reasons that make this particular fitness landscape a difficult one. In this paper we statistically quantify in various ways the degree of difficulty of searching the majority CA landscape.

The paper proceeds as follows. The next section summarizes some known facts about CAs for the density task. A description of its fitness landscape follows, focusing on the hardness and neutrality aspects. Next we identify and analyze a particular subspace of the problem search space called the Olympus. Finally, we present our conclusions and hints to further works and open questions. 


\section{The Majority Problem}

The density task is a prototypical distributed computational problem for CAs. For a finite CA of size $N$ it is defined as follows. Let $\rho_{0}$ be the fraction of $1 \mathrm{~s}$ in the initial configuration (IC) $\mathbf{s}_{0}$. The task is to determine whether $\rho_{0}$ is greater than or less than $1 / 2$. In this version, the problem is also known as the majority problem. If $\rho_{0}>1 / 2$ then the CA must relax to a fixed-point configuration of all 1 's that we indicate as $(1)^{N}$; otherwise it must relax to a fixed-point configuration of all 0 's, noted $(0)^{N}$, after a number of time steps of the order of the grid size $N$. Here $N$ is set to 149 , the value that has been customarily used in research on the density task (if $N$ is odd one avoids the case $\rho_{0}=0.5$ for which the problem is undefined).

This computation is trivial for a computer having a central control. Indeed, just scanning the array and adding up the number of, say, 1 bits will provide the answer in $O(N)$ time. However, it is nontrivial for a small radius one-dimensional CA since such a CA can only transfer information at finite speed relying on local information exclusively, while density is a global property of the configuration of states. It has been shown that the density task cannot be solved perfectly by a uniform, two-state CA with finite radius [2].

The lack of a perfect solution does not prevent one from searching for imperfect solutions of as good a quality as possible. In general, given a desired global behavior for a CA (e.g., the density task), it is extremely difficult to infer the local CA rule that will give rise to the emergence of the computation sought. This is because of the possible nonlinearities and large-scale collective effects that cannot in general be predicted from the sole local CA updating rule, even if it is deterministic. Since exhaustive evaluation of all possible rules is out of the question except for elementary $(d=1, r=1)$ and perhaps radius-two automata, one possible solution consists in using evolutionary algorithms, as first proposed by Packard in [7] and further developed by Mitchell et al. [3,6].

The standard performance of the best rules (with $r=3$ ) found at the end of the evolution is defined as the fraction of correct classifications over $n=10^{4}$ randomly chosen ICs. The ICs are sampled according to a binomial distribution (i.e., each bit is independently drawn with probability $1 / 2$ of being 0 ).

Mitchell and coworkers performed a number of studies on the emergence of synchronous CA strategies for the density task (with $N=149$ ) during evolution [6.3]. Their results are significant since they represent one of the few instances where the dynamics of emergent computation in complex, spatially extended systems can be understood. As for the evolved CAs, it was noted that, in most runs, the GA found unsophisticated strategies that consisted in expanding sufficiently large blocks of adjacent 1 s or 0s. This "block-expanding" strategy is unsophisticated in that it mainly uses local information to reach a conclusion. As a consequence, only those IC that have low or high density are classified correctly since they are more likely to have extended blocks of 1 s or 0 s. These CAs have a performance around 0.6. A few runs yielded more sophisticated CAs with performance (around 0.77) on a wide distribution of ICs. However, high-performance automata have evolved only nine times out of 300 runs of the 
genetic algorithm. This clearly shows that the search space is a very difficult one, even there exists some recent works on coevolutionary algorithm [8] which able to find a number of "block expanding" strategies.

These sophisticated strategies rely on traveling signals ("particles") that transfer spatial and temporal information about the density in local regions through the lattice, and have been quantitatively described with a framework known as "computational mechanics" 9, 10]. The GKL rule 11] is hand-coded but its behavior is similar to that of the best solutions found by evolution. Das and Davis solutions are two other good solutions that have been found by hand [6]. Other researchers have been able to artificially evolve a better CA $(A B K)$ by using genetic programming CAs $(C o e 1$ and $C o e 2)$ by using a coevolutionary algorithm. Their coevolved CA has performance about 0.86 , which is the best result known to date. We call the six best local optima known, with a standard performance over 0.81 , the blok (tab. 11).

In the next section we present a study of the overall fitness landscape, while section 4.2 concentrates on the structure of the landscape around the blok.

Table 1. Description in hexadecimal and standard performance of the 6 previously known best rules (blok) computed on sample size of $10^{4}$.

\begin{tabular}{|c|c|}
\hline GKL 0.815 & Das 0.823 \\
005F005F005F005F005FFF5F005FFF5F & 009F038F001FBF1F002FFB5F001FFF1F \\
\hline Davis 0.818 & ABK 0.824 \\
070007FF0F000FFF0F0007FF0F310FFF & 050055050500550555FF55FF55FF55FF \\
\hline Coe1 0.851 & Coe2 0.860 \\
011430D7110F395705B4FF17F13DF957 & 1451305C0050CE5F1711FF5F0F53CF5F \\
\hline
\end{tabular}

\section{Fitness Landscape and Neutrality of the Majority Task}

First we recall a few fundamental concepts about fitness landscapes 12]. A fitness landscape is a triplet $(\mathcal{S}, \mathcal{V}, f)$ such that : $\mathcal{S}$ is the set of potential solutions, $\mathcal{V}: \mathcal{S} \rightarrow 2^{\mathcal{S}}$ is the neighborhood function which associates to each solution $s \in \mathcal{S}$ a set of neighbor solutions $\mathcal{V}(s) \subset S, f: \mathcal{S} \rightarrow \mathbb{R}$ is the fitness function which associates a real number to each solution.

Within the framework of metaheuristic by local search, the local operators allow to define the neighborhood $\mathcal{V}$. If the metaheuristic only uses one operator $o p$, the neighborhood of a solution $s$ is often defined as $\mathcal{V}(s)=\left\{s^{\prime} \in \mathcal{S} \mid s^{\prime}=\right.$ $o p(s)\}$. If more than one operator are used, it is possible to associate one fitness landscape to each operator or to define the set of neighbors as the set of solutions obtained by one of the operators. A neighborhood could be associated to a distance; for example, in the field of genetic algorithms, when the search space is 
the set of bit strings of fixed size, the operator which change the value of one bit defines the neighborhood. Thus, two solutions are neighbors if their Hamming distance is equal to 1 .

The notion of neutrality has been suggested by Kimura [13] in his study of the evolution of molecular species. According to this view, most mutations are either neutral (their effect on fitness is small) or lethal. In the analysis of fitness landscapes, the notion of neutral mutation appears to be useful [12]. Let us thus define more precisely the notion of neutrality for fitness landscapes.

A test of neutrality is a predicate isNeutral $: S \times S \rightarrow\{$ true, false $\}$ that assigns to every $\left(s_{1}, s_{2}\right) \in S^{2}$ the value true if there is a small difference between $f\left(s_{1}\right)$ and $f\left(s_{2}\right)$.

For example, usually isNeutral $\left(s_{1}, s_{2}\right)$ is true if $f\left(s_{1}\right)=f\left(s_{2}\right)$. In that case, $i s N$ eutral is an equivalence relation. Other useful cases are $i s N$ eutral $\left(s_{1}, s_{2}\right)$ is true if $\left|f\left(s_{1}\right)-f\left(s_{2}\right)\right| \leq 1 / M$ with $M$ is the population size. When $f$ is stochastic, isNeutral $\left(s_{1}, s_{2}\right)$ is true if $\left|f\left(s_{1}\right)-f\left(s_{2}\right)\right|$ is under the evaluation error.

For every $s \in S$, the neutral neighborhood of $s$ is the set $\mathcal{V}_{\text {neut }}(s)=\left\{s^{\prime} \in\right.$ $\mathcal{V}(s) \mid$ isNeutral $\left.\left(s, s^{\prime}\right)\right\}$ and the neutral degree of $s$, noted $n D e g(s)$ is the number of neutral neighbors of $s, n \operatorname{Deg}(s)=\sharp\left(\mathcal{V}_{\text {neut }}(s)-\{s\}\right)$.

A fitness landscape is neutral if there are many solutions with high neutral degree. In this case, we can imagine fitness landscapes with some plateaus called neutral networks. There is no significant difference of fitness between solutions on neutral networks and the population drifts around on them.

A neutral walk $W_{\text {neut }}=\left(s_{0}, s_{1}, \ldots, s_{m}\right)$ is a walk where for all $i \in[0, m-1]$, $s_{i+1} \in \mathcal{V}\left(s_{i}\right)$ and for all $(i, j) \in[0, m]^{2}$, isNeutral $\left(s_{i}, s_{j}\right)$ is true.

A Neutral Network, denoted $N N$, is a graph $G=(V, E)$ where the set $V$ of vertices is the set of solutions belonging to $S$ such that for all $s$ and $s^{\prime}$ from $V$ there is a neutral walk $W_{\text {neut }}$ belonging to $V$ from $s$ to $s^{\prime}$, and two vertices are connected by an edge of $E$ if they are neutral neighbors.

\subsection{Statistical Measures of neutrality}

H. Rosé et al. 14 develop the density of states approach (DOS) by plotting the number of sampled solutions in the search space with the same fitness value. Knowledge of this density allows to evaluate the performance of random search or random initialization of metaheuristics. DOS gives the probability of having a given fitness value when a solution is randomly chosen. The tail of the distribution at optimal fitness value gives a measure of the difficulty of an optimization problem: the faster the decay, the harder the problem.

To study the neutrality of fitness landscapes, we should be able to measure and describe a few properties of $N N$. The following quantities are useful. The size $\sharp N N$ i.e., the number of vertices in a $N N$, the diameter, which is the maximum distance between two solutions belonging to $N N$. The neutral degree distribution of solutions is the degree distribution of the vertices in a $N N$. Together with the size and the diameter, it gives information which plays a role in the dynamics of metaheuristic [15]. Another way to describe $N N$ is given by the autocorrelation of neutral degree along a neutral random walk 16. At each 
step $s_{i}$ of the walk, one neutral solution $s_{i+1} \in \mathcal{V}\left(s_{i}\right)$ is randomly chosen such as $\forall j \leq i$, isNeutral $\left(s_{j}, s_{j}\right)$ is true. From neutral degree collected along this neutral walk, we computed its autocorrelation. The autocorrelation measures the correlation structure of a $N N$. If the correlation is low, the variation of neutral degree is low ; and so, there is some areas in $N N$ of solutions which have nearby neutral degrees.

\section{Neutrality in the Majority Problem landscape}

In this work we use a performance measure, the standard performance defined in section 2, which is based on the fraction of $n$ initial configurations that are correctly classified from one sample. Standard performance is a hard measure because of the predominance in the sample of ICs close to 0.5 and it has been typically employed to measure a CA's capability on the density task.

The error of evaluation leads us to define the neutrality of the landscape. The standard performance cannot be known perfectly due to random variation of samples of ICs. The ICs are chosen independently, so the fitness value $f$ of a solution follows a normal law $\mathcal{N}\left(f, \frac{\sigma(f)}{\sqrt{n}}\right)$, where $\sigma$ is the standard deviation of sample of fitness $f$, and $n$ is the sample size. For binomial sample, $\sigma^{2}(f)=f(1-f)$, the variance of Bernouilli trial. Thus two neighbors $s$ and $s^{\prime}$ are neutral neighbors (isNeutral $\left(s, s^{\prime}\right)$ is true) if a t-test accepts the hypothesis of equality of $f(s)$ and $f\left(s^{\prime}\right)$ with 95 percent of confidence. The maximum number of fitness values statistically different for standard performance is 113 for $n=10^{4}, 36$ for $n=10^{3}$ and 12 for $n=10^{2}$.

\subsection{Analysis of the Full Landscape}

Density Of States. It has proved difficult to obtain information on the Majority Problem landscape by random sampling due to the large number of solutions with zero fitness. From $4.10^{3}$ solutions using the uniform random sampling technique, 3979 solutions have a fitness value equal to 0 . Clearly, the space appears to be a difficult one to search since the tail of the distribution to the right is non-existent. Figure 3 a shows the DOS obtained using the Metropolis-Hastings technique for importance sampling. For the details of the techniques used to sample high fitness values of the space, see [17]. This time, over the $4.10^{3}$ solutions sampled, only 176 have a fitness equal to zero, and the DOS clearly shows a more uniform distribution of rules over many different fitness values. It is important to remark a considerable number of solutions sampled with a fitness approximately equal to 0.5 . Furthermore, no solution with a fitness value superior to 0.55 has been sampled.

Computational costs do not allow us to analyse many neutral networks. In this section we analyse two important large neutral networks $(N N)$. A large number of CAs solve the majority density problem on only half of ICs because 
they converge nearly always on the final configuration $(O)^{N}$ or $(1)^{N}$ and thus have performance about 0.5. Mitchell et al. 3] call these "default strategies" and notice that they are the first stage in the evolution of the population before jumping to higher performance values associated to "block-expanding" strategies (see section 2). We will study this large $N N$, denoted $N N_{0.5}$ around standard performance 0.5 to understand the link between $N N$ properties and GA evolution. The other $N N$, denoted $N N_{0.76}$, is the $N N$ around fitness 0.7645 which contains one neighbor of a CA found by Mitchell et al. The description of this "high" $N N$ could give clues as how to "escape" from $N N$ toward even higher fitness values.

Diameter. In our experiments, we perform 5 neutral walks on $N N_{0.5}$ and 19 on $N N_{0.76}$. Each neutral walk has the same starting point on each $N N$. We try to explore the $N N$ by strictly increasing the Hamming distance from the starting solution at each step of the walk. The neutral walk stops when there is no neutral step that increases distance. The maximum length of walk is thus 128 . On average, the length of neutral walks on $N N_{0.5}$ is 108.2 and 33.1 on $N N_{0.76}$. The diameter of $N N_{0.5}$ is thus larger than the one of $N N_{0.76}$.

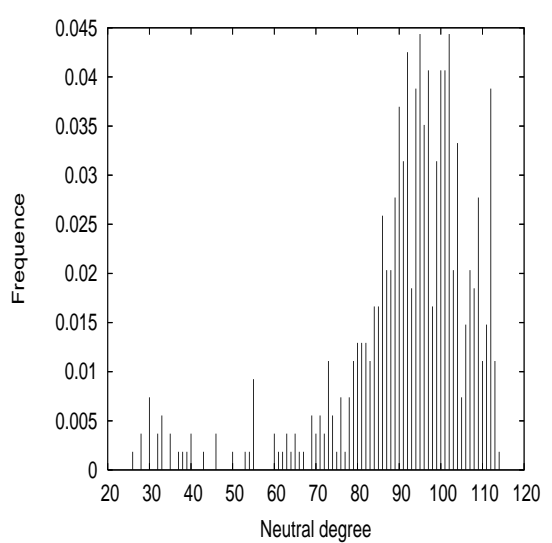

(a)

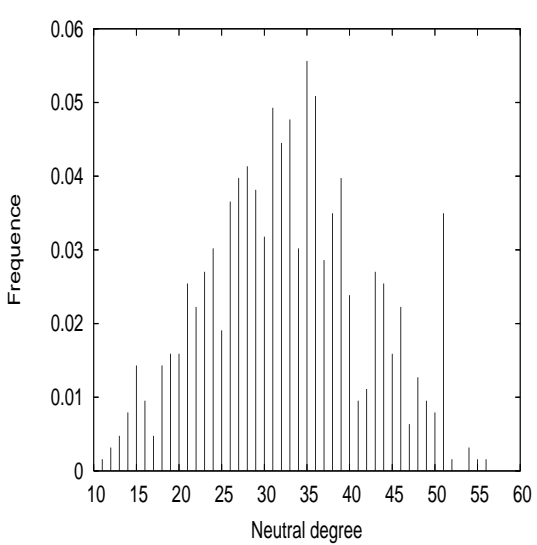

(b)

Fig. 1. Distribution of Neutral Degree along all neutral walks on $N N_{0.5}$ in (a) and $N N_{0.76}$ in (b).

Neutral Degree Distribution. Figure 11 shows the distribution of neutral degree collected along all neutral walks. The distribution is close to normal for $N N_{0.76}$. For $N N_{0.5}$ the distribution is skewed and approximately bimodal with a strong peak around 100 and a small peak around 32. The average of neutral degree on $N N_{0.5}$ is 91.6 and standard deviation is 16.6 ; on $N N_{0.76}$, the average is 32.7 
and the standard deviation is 9.2. The neutral degree for $N N_{0.5}$ is very high : $71.6 \%$ of neighbors are neutral neighbors. For $N N_{0.76}$, there is $25.5 \%$ of neutral neighbors. It can be compared to the average neutral degree of the neutral $N K q$ landscape with $N=64, K=2$ and $q=2$ which is $33.3 \%$.

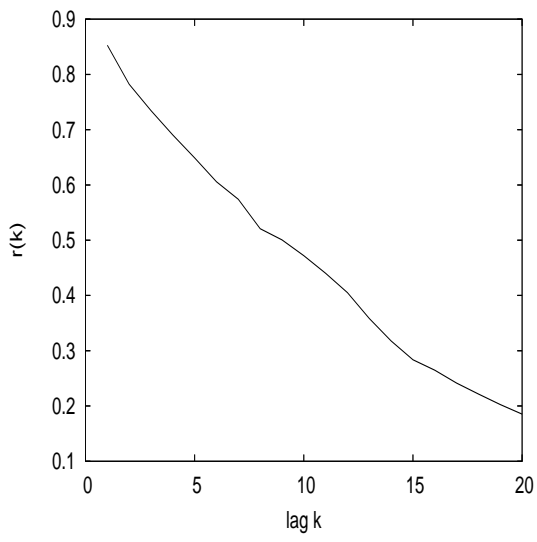

(a)

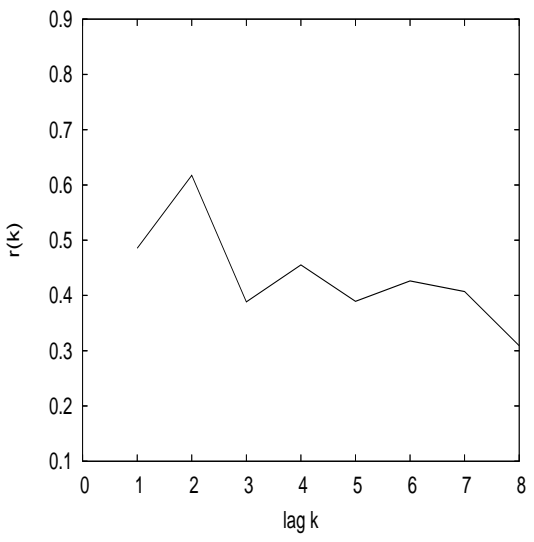

(b)

Fig. 2. Estimation of the autocorrelation function of neutral degrees along neutral random walks for $N N_{0.5}$ (a) and for $N N_{0.76}$ (b).

Autocorrelation of Neutral Degree. Figure 2 gives an estimation of the autocorrelation function $\rho(k)$ of neutral degree of the neutral networks. The autocorrelation function is computed for each neutral walk and the estimation $r(k)$ of $\rho(k)$ is given by the average of $r_{i}(k)$ over all autocorrelation functions. For both $N N$, there is correlation. The correlation is higher for $N N_{0.5}(r(1)=0.85)$ than for $N N_{0.76}(r(1)=0.49)$. From the autocorrelation of the neutral degree, one can conclude that the neutral network topology is not completely random, since otherwise correlation should have been nearly equal to zero. Moreover, the variation of neutral degree is smooth on $N N$; in other words, the neighbors in $N N$ have nearby neutral degrees. So, there is some area where the neutral degree is homogeneous.

This study give us a better description of Majority fitness landscape neutrality which have important consequence on metaheuristic design. The neutral degree is high. Therefore, the selection operator should take into account the case of equality of fitness values. Likewise the mutation rate and population size should fit to this neutral degree in order to find rare good solutions outside $N N$ [18. For two potential solutions $x$ and $y$ on $N N$, the probability $p$ that at least one solution escaped from $N N$ is $P(x \notin N N \cup y \notin N N)=P(x \notin N N)+P(y \notin$ $N N)-P(x \notin N N \cap y \notin N N)$. This probability is higher when solutions $x$ and 
$y$ are far due to the correlation of neutral degree in $N N$. To maximize the probability of escaping $N N$ the distance between potential solutions of population should be as far as possible on $N N$. The population of an evolutionary algorithm should spread over $N N$.

\subsection{Study on the Olympus Landscape}

In this section we show that there are many similarities inside the blok (see section 2), and we use this feature to define what we have named the Olympus Landscape, a subspace of the full landscape in which good solutions are found. Next, we study the relevant properties of this subspace. Before defining the Olympus we study the two natural symmetries of the majority problem.

The states 0 and 1 play the same role in the computational task; so flipping bits in the entry of a rule and in the result have no effect on performance. In the same way, CAs can compute the majority problem according to right or left direction without changing performance. We denote $S_{01}$ and $S_{r l}$ respectively the corresponding operator of $0 / 1$ symmetry and right/left symmetry. Let $x=$ $\left(x_{0}, \ldots, x_{\lambda-1}\right) \in\{0,1\}^{\lambda}$ be a solution with $\lambda=2^{2 r+1}$. The $0 / 1$ symmetric of $x$ is $S_{01}(x)=y$ where for all $i, y_{i}=1-x_{\lambda-i}$. The right/left symmetric of $x$ is

$S_{r l}(x)=y$ where for all $i, y_{i}=x_{\sigma(i)}$ with $\sigma\left(\sum_{j=0}^{\lambda-1} 2^{n_{j}}\right)=\sum_{j=0}^{\lambda-1} 2^{\lambda-1-n_{j}}$. The operators are commutative: $S_{r l} S_{01}=S_{01} S_{r l}$. From the 128 bits, 16 are invariant by $S_{r l}$ and none by $S_{01}$.

Two optima from the blok could be distant whereas some of theirs symmetrics are closer. Here the idea is to choose for each blok one symmetric in order to broadly maximize the number of joint bits.

The optima GKL, Das, Davis and ABK have 2 symmetrics only because symmetrics by $S_{01}$ and $S_{r l}$ are equal. The optima Coe1 and Coe2 have 4 symmetrics. So, there are $2^{4} .4^{2}=256$ possible sets of symmetrics. Among these sets, we establish the maximum number of joint bits which is possible to obtain is 51 . This "optimal" set contains the six Symmetrics of Best Local Optima Known (blok') which are GKL' $=$ GKL, Das ${ }^{\prime}=$ Das, Davis $^{\prime}=S_{01}$ (Davis), ABK $^{\prime}=S_{01}(\mathrm{ABK})$, Coe $1^{\prime}=$ Coe1 and $\mathrm{Coe} 2^{\prime}=S_{r l}(\mathrm{Coe} 2)$.

The Olympus Landscape is defined from the blok' as the subspace of dimension 77 defined by the string $S^{\prime}$ :

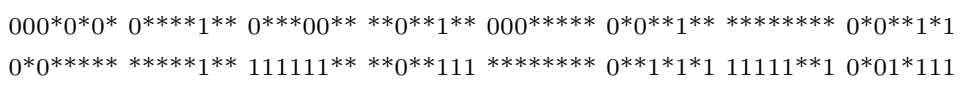

Density Of States. The DOS is more favorable in the Olympus with respect to the whole search space by sampling the space uniformly at random, only $28.6 \%$ solutions have null fitness in the random sample. Figure 3 a shows the DOS on the Olympus which has been obtained by sampling with the Metropolis-Hastings method. Only $0.3 \%$ solutions have null fitness value in this sample, although the tail of the distribution is fast-decaying beyond fitness value 0.5 the highest solution for $\mathrm{M}-\mathrm{H}$ is 0.68 . The DOS thus justifies the favours to concentrate the search in the Olympus landscape. 


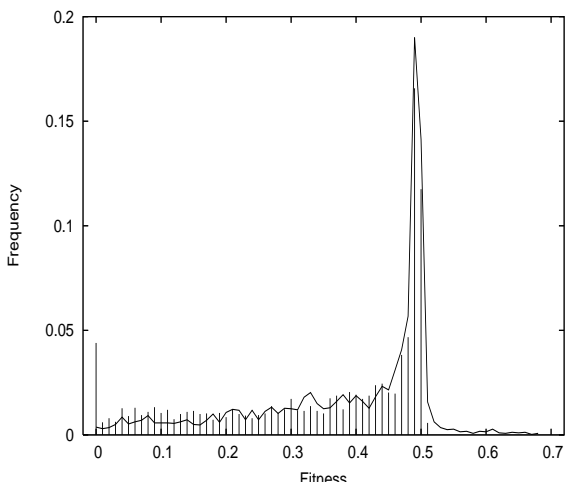

(a)

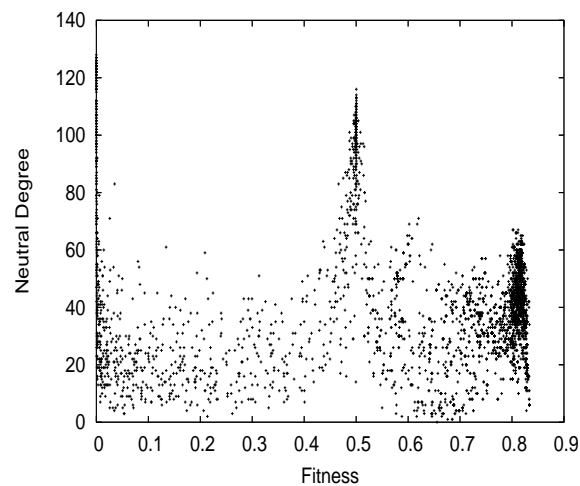

(b)

Fig. 3. (a) DOS using Metropolis-Hasting technique to sample the whole space (impulse) and the Olympus Landscape (line). (b) Neutral degree on Olympus as a function of the performance.

Neutral Degree. The figure 3-b gives the neutral degree of solutions from Olympus as a function of their performance. The solutions below performance 0.5 are randomly chosen in Olympus. The solutions over performance 0.5 are sampled with 2 runs of a GA during $10^{3}$ generations. This GA is based on GA defined by Mitchell [3] where the operators are restricted to Olympus subspace and the selection is a tournament selection of size 2 taking into account the neutrality. This GA allows to discover a lot of solutions between 0.80 and 0.835 and justified the useful of Olympus ${ }^{4}$. Two important $N N$ are located around fitnesses 0 and 0.5 where the neutral degree is over 70 . For solutions over 0.5 , the average of neutral degree is 37.6 which is a high neutral degree.

\section{Discussion and Conclusion}

The landscape has a considerable number of points with performance 0 or 0.5 which means that investigations based on sampling techniques on the whole landscape are unlikely to give good results. The neutrality of the landscape is high, and the neutral network topology is not completely random. Exploiting similarities between the six best rules and symmetries in the landscape, we have defined the Olympus landscape as a subspace of the Majority problem landscape. This subspace have less solutions with performance 0 and it is easy to find solutions over 0.80 with a simple GA. We have shown that the neutrality of landscape is high even for solution over 0.5.

\footnotetext{
${ }^{4}$ Over 50 runs, average performances are 0.832 with standard deviation 0.006 which is higher than $0.80_{0.02}$ of coevolutionary algorithm of Pagie [8]
} 


\section{References}

1. Wolfram, S.: A New Kind of Science. Wolfram Media (2002)

2. Land, M., Belew, R.K.: No perfect two-state cellular automata for density classification exists. Physical Review Letters 74 (1995) 5148-5150

3. Mitchell, M., Crutchfield, J.P., Hraber, P.T.: Evolving cellular automata to perform computations: Mechanisms and impediments. Physica D 75 (1994) 361-391

4. Andre, D., Bennett III, F.H., Koza, J.R.: Discovery by genetic programming of a cellular automata rule that is better than any known rule for the majority classification problem. In Koza, J.R., Goldberg, D.E., Fogel, D.B., Riolo, R.L., eds.: Genetic Programming 1996: Proceedings of the First Annual Conference, Cambridge, MA, The MIT Press (1996) 3-11

5. Juillé, H., Pollack, J.B.: Coevolutionary learning: a case study. In: ICML '98 Proceedings of the Fifteenth International Conference on Machine Learning, San Francisco, CA, Morgan Kaufmann (1998) 251-259

6. Crutchfield, J.P., Mitchell, M., Das, R.: Evolutionary design of collective computation in cellular automata. In Crutchfield, J.P., Schuster, P., eds.: Evolutionary Dynamics: Exploring the Interplay of Selection, Accident, Neutrality, and Function. Oxford University Press, Oxford, UK (2003) 361-411

7. Packard, N.H.: Adaptation toward the edge of chaos. In Kelso, J.A.S., Mandell, A.J., Shlesinger, M.F., eds.: Dynamic Patterns in Complex Systems. World Scientific, Singapore (1988) 293-301

8. Pagie, L., Mitchell, M.: A comparison of evolutionary and coevolutionary search. In Belew, R.K., Juillè, H., eds.: Coevolution: Turning Adaptive Algorithms upon Themselves, San Francisco, California, USA (2001) 20-25

9. Das, R., Mitchell, M., Crutchfield, J.P.: A genetic algorithm discovers particlebased computation in cellular automata. In Davidor, Y., Schwefel, H.P., Männer, R., eds.: PPSN III. Volume 866 of LNCS., Springer-Verlag (1994) 344-353

10. Hanson, J.E., Crutchfield, J.P.: Computational mechanics of cellular automata: An example. Technical Report 95-10-95, Santa Fe Institute Working Paper (1995)

11. Gacs, P., Kurdyumov, G.L., Levin, L.A.: One-dimensional uniform arrays that wash out finite islands. Problemy Peredachi Informatsii 14 (1978) 92-98

12. Reidys, C.M., Stadler, P.F.: Neutrality in fitness landscapes. Applied Mathematics and Computation 117 (2001) 321-350

13. Kimura, M.: The Neutral Theory of Molecular Evolution. Cambridge University Press, Cambridge, UK (1983)

14. Rosé, H., Ebeling, W., Asselmeyer, T.: The density of states - a measure of the difficulty of optimisation problems. In: Parallel Problem Solving from Nature. (1996) 208-217

15. Van Nimwegen, E., Crutchfield, J., Huynen, M.: Neutral evolution of mutational robustness. In: Proc. Nat. Acad. Sci. USA 96. (1999) 9716-9720

16. Bastolla, U., Porto, M., Roman, H.E., Vendruscolo, M.: Statiscal properties of neutral evolution. Journal Molecular Evolution 57 (2003) 103-119

17. Vanneschi, L., Clergue, M., Collard, P., Tomassini, M., Verel, S.: Fitness clouds and problem hardness in genetic programming. In: Proceedings of GECCO'04. LNCS, Springer-Verlag (2004)

18. Barnett, L.: Netcrawling - optimal evolutionary search with neutral networks. In: Proceedings of the 2001 Congress on Evolutionary Computation CEC2001, COEX, World Trade Center, 159 Samseong-dong, Gangnam-gu, Seoul, Korea, IEEE Press (2001) 30-37 